\title{
Etude technico-économique d'un système hybride (aérogénérateur et moteur diesel) pour la production d'électricité
}

\author{
Laarej MERAD ${ }^{1, *}$, Boumédiène BENYOUCEF ${ }^{1}$ ef Jean Pierre CHARLES ${ }^{2}$
}

'Centre universitaire Mustapha STAMBOULI de Mascara, Unité de recherche "I Matériaux et énergies renouvelables II, Université de Tlemcen, Faculté des Sciences, Département de Physique, B.P 119 Tlemcen 13000, Algérie

${ }^{2}$ CEEA - MOPS - CLOES, Supélec, 2, rue Edouard Belin, 57070 Metz, France.

(Reçu le 22 Février 2006, accepté le 10 Mai 2006)

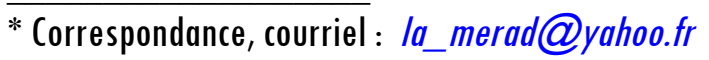

\section{Résumé}

Dans le présent article on s'intéresse à l'étude technico-économique d'un système hybride (aérogénérateur et moteur Diesel) sur le site de Tlemcen en utilisant les données de mesure des stations de l'ONM (Office National de Météorologie). On évalue la puissance moyenne annuelle disponible sur le site, la puissance moyenne annuelle fournie par l'aérogénérateur et le moteur Diesel ainsi que la détermination du nombre de foyers pouvant être alimentés en énergie électrique. Cette étude se poursuit par un aspect économique car le développement des aérogénérateurs et les moteurs Diesel relèvent du domaine technique mais aussi du domaine économique. Pour que la production de l'énergie électrique soit viable, il faut que son coût de revient soit moins élevé que celui de l'énergie électrique produite par un moteur Diesel ou une turbine; le rendement et le coût étant deux facteurs liées entre eux.

La viabilité de l'installation serait peu intéressante pour un rendement et un coût de revient élevés ou pour un rendement et un coût de revient faibles.

Mots-Clés : Aérogénérateur, moteur Diesel, électricité, puissance, économie.

\section{Abstract}

Technico-economic study of a hybrid system (Aerogenerator and Diesel engine) for the electricity production

In this article one is interested in the technico-economic study of a hybrid system (aerogenerator and Diesel engine) on the site of Tlemcen by using the data of measurement of the stations of the ONM (National office of Meteorology), one evaluates the annual aver- 
age power available on the site as well as the annual average power provided by the aerogenerator and the diesel engine thus the determination of the number of hearths which can be to feed in electric power finally this study continues with an economic aspect because the development of the aerogenerators and the diesel engines come under the technical field but also of the economic field, so that the production of electrical energy is viable that is to say less low than that of that produced by a Diesel engine or a turbine, the output and the cost are two factors dependent between them.

The viability of the installation would be little interest for an output and a cost of high cost or for an output and a weak cost of cost.

Keywords: Aerogenerator, Diesel engine, electricity, power, economy.

\section{Introduction}

Les sources d'énergie non renouvelables (Charbon, Pétrole, Gaz et Uranium) s'épuisent au fur et à mesure de leur exploitation contrairement à l'éolien, au solaire, à l'hydraulique et à la biomasse qui seront disponibles pendant encore environs 5 milliards d'années. Or la consommation énergétique mondiale de même que la production d'électricité est largement dominée par les énergies fossiles qui représentent plus de $85 \%$ de l'énergie primaire.

L'éolien, qui correspond à la transformation en énergie mécanique de $1 \%$ de l'énergie reçue sur terre, est en plein essor, mais produit un kWh dont le prix reste 2 à 4 fois supérieur à celui du nucléaire.

Le schéma le plus simple d'un système énergétique est celui que l'homme exploite depuis des millénaires en utilisant le bois pour cuire ces aliments ou se chauffer et la force animale ou la force du vent pour moudre le grain ou se déplacer. D'une énergie primaire puisée dans la nature, le bois et le vent en l'occurrence, l'homme tire une énergie utile qui correspond à un service, le chauffage ou le mouvement. Ce n'est qu'à partir de $18^{\mathrm{e}}$ siècle que le concept d'énergie s'est dégagé peu à peu. L'énergie est devenue un moteur essentiel de l'économie.

Le coût de production du kilowattheure a toujours fait l'objet d'une analyse approfondie. Dans notre cas l'énergie éolienne et un moteur Diesel, autrefois sources d'énergie mécanique, aujourd'hui sources d'électricité. La maturité technologique de la filière éolienne rend aujourd'hui cette source d'énergie électrique rentable.

Comme ressource d'appoint pour alléger la charge d'un réseau électrique tout particulièrement en hiver lorsque la consommation augmente fortement.

Comme source d'alimentation des sites isolés pour être raccordés au réseau à un prix compétitif. 
Comme source d'alimentation des sites présentant des contraintes climatiques ou d'accessibilité.

Notre étude est menée pour quantifier la puissance énergétique disponible et la densité de puissance moyenne annuelle récupérable sur le site de TLEMCEN. C'est dans un site admirable, au milieu d'un écrin de verdure et par plus de $800 \mathrm{~m}$ d'altitude, qu'on dresse la ville de Tlemcen. D'abord sa position sur une haute plaine de piémont, au carrefour des grandes routes reliant l'Ouest algérien au Maroc et le tell du Sahara, l'a prédisposée à servir de lieu d'échanges entre des économies complémentaires rurale et citadine, agricole et pastorale. La proximité de la mer a pour effet d'atténuer les influences desséchantes du continent et de donner à la région, un climat tempéré, d'une douceur toute méditerranéenne et parce qu'elle reçoit des pluies abondantes, son territoire qui correspond au bassin de la Tafna avec ses nombreux influents et ses sources.

En s'appuyant sur le modèle mathématique de la distribution de WEIBULL, tout système de conversion de l'énergie éolienne fonctionne dans des conditions optimales après avoir été dimensionné selon les caractéristiques du site. Les paramètres qui caractérisent un aérogénérateur sont la puissance nominale $P_{n}$, la vitesse de démarrage $V_{d}$, la vitesse nominale $V_{n}$, et la vitesse de coupure $V_{c}$.

L'estimation de la puissance moyenne annuelle de sortie d'un aérogénérateur à partir de la loi de distribution de WEIBULL permet d'apprécier les possibilités d'alimentation des locaux des sites isolés.

\section{Modélisation mathématique}

La distribution de WEIBULL nous renseigne sur la répartition de la vitesse du vent (vitesse moyenne, vitesse cubique moyenne et la densité de probabilité de la vitesse du vent).

Généralement les vitesses du vent sont mesurées à une hauteur de 10 mètres a.d.s (au dessus du sol) et ce dans les stations de l'ONM (Office National de la Météorologie).

La pression variant à chaque instant, on observe des fluctuations de la vitesse du vent qui sont amplifiées par le phénomène de turbulence.

Le modèle mathématique de WEIBULL est caractérisé par les paramètres de forme $k$ et d'échelle $C$.

La densité de probabilité de la puissance s'écrit sous la forme suivante :

$$
f(V)=\left(\frac{k}{C}\right) \cdot\left(\frac{V}{C}\right)^{k-1} \cdot \exp \left[-\left(\frac{V}{C}\right)\right]^{k}
$$

Pour déterminer les caractéristiques de la vitesse du vent à des hauteurs supérieures à 
10 mètres a.d.s on procède à l'extrapolation verticale des paramètres de WEIBULL, en utilisant la méthode de JUSTUS $[1,2,3]$ qui donne la loi de puissance modifiée par le facteur d'échelle $C_{1}$ et de forme $k_{1}$.a 10 mètres a.d.s $C_{2}$ et $k_{2}$ a 25 mètres a.d.s.

$$
C_{2}=C_{1} \cdot\left(\frac{Z_{2}}{Z_{1}}\right)^{(\alpha k)}
$$

0ù

$$
\begin{aligned}
& \alpha k=\frac{1}{\ln \left(\frac{\sqrt{Z_{1} \cdot Z_{2}}}{Z_{0}}\right)}-0.0881 \cdot \ln \left(\frac{C_{1}}{6}\right) \\
& k_{2}=\frac{k_{1}}{\left[1-0.0881 \cdot \ln \left(\frac{Z_{2}}{Z_{1}}\right)\right]}
\end{aligned}
$$

La vitesse moyenne du vent est donnée par la distribution de WEIBULL [4] :

$$
\bar{V}=\int_{0}^{\infty} f(V) \cdot d V=C \cdot \Gamma\left(1+\frac{1}{k}\right)
$$

Où $\Gamma$ est la fonction GAMMA. La fonction GAMMA est définie par la relation suivante :

$$
\Gamma(x)=\int_{0}^{\infty} \exp (-t) \cdot t^{x-1} \cdot d t \quad \text { avec } \quad x>0
$$

La vitesse cubique moyenne du vent est donnée par la relation suivante :

$$
\left\langle V^{3}\right\rangle=\int_{0}^{\infty} V^{3} \cdot P(V) \cdot d V=C^{3} \cdot \Gamma\left(1+\frac{3}{k}\right)
$$

La densité de puissance moyenne annuelle [12] récupérable $\langle P\rangle_{\text {moy }}$ exprimée en $\mathrm{kWh} / \mathrm{m}^{2}$.an se calcule à partir de la formule suivante :

$$
\begin{aligned}
\langle P\rangle_{\text {moy }}=T & \cdot\left(P_{i}\right)(7) \\
& \text { avec : } P_{i}=0.37 \cdot\left\langle V^{3}{ }_{i}\right\rangle
\end{aligned}
$$

Où : $T$ : L'échelle de temps à une année $T=365.24=8760$ heures/an.

Enfin la densité de puissance moyenne annuelle récupérable est :

$$
\langle P\rangle_{\text {moy }}=32.5 \cdot\left\langle V^{3}{ }_{i}\right\rangle
$$




\section{2-1. Puissance moyenne annuelle fournie par un aérogénérateur}

Chaque système de conversion d'énergie éolienne est caractérisé par $[4,5]$ :

- La vitesse de démarrage (Puissance nulle).

- La vitesse nominale ou maximale (puissance maximale).

- La vitesse de coupure (puissance constante, pour éviter la détérioration du matériel si la vitesse augmente).

La puissance moyenne annuelle fournie par un aérogénérateur est donnée sous la forme suivante :

$$
\bar{P}=\int_{0}^{\infty} P(V) \cdot f(V) \cdot d V
$$

La variation de la puissance utile fournie est exprimée à l'aide du système d'équations suivant, appelé aussi loi quadratique $[4,5]$.

$$
P(V)=\left\{\begin{array}{llll}
0 & \alpha+\beta . V+\gamma . V^{2} & \text { si } & \\
& \text { si } & V_{d}<V<V_{d} \\
\boldsymbol{P}_{n} & \text { si } & V_{n}<V<V_{c} \\
0 & \text { si } & V>V_{c}
\end{array}\right.
$$

avec $\alpha, \beta$ et $\gamma$ déterminés par les conditions suivantes:

$$
\left\{\begin{array}{l}
\alpha+\beta \cdot V_{d}+\gamma \cdot V_{d}^{2}=0 \\
\alpha+\beta \cdot V_{n}+\gamma \cdot V_{n}^{2}=P_{n} \\
\alpha+\beta \cdot\left(V_{d}+V_{n}\right)+\gamma \cdot\left(V_{d}+V_{n}\right)^{3}=P_{n} \cdot\left(\frac{\left(V_{d}+V_{n}\right)}{V_{n}}\right)^{3}
\end{array}\right.
$$

L'intégrale (10) va s'écrire sous la forme suivante :

$$
\bar{P}=\int_{V_{d}}^{V_{n}}\left(\alpha+\beta \cdot V+\gamma \cdot V^{2}\right) \cdot \frac{k_{1}}{C_{1}} \cdot\left(\frac{V}{C_{1}}\right)^{k_{1}-1} \cdot \exp \left(-\left(\frac{V}{C_{1}}\right)^{k_{1}}\right) \cdot d V+\int_{V_{n}}^{V_{c}} P_{n} \cdot \frac{k_{1}}{C_{1}} \cdot\left(\frac{V}{C_{1}}\right)^{k_{1}-1} \cdot \exp \left(-\left(\frac{V}{C_{1}}\right)^{k_{1}}\right) \cdot d V
$$

L'intégrale $I_{2}$ :

$$
I_{2}=\int_{V_{n}}^{V_{c}} P_{n} \cdot \frac{k_{1}}{C_{1}} \cdot\left(\frac{V}{C_{1}}\right)^{k_{1}-1} \cdot \exp \left(-\left(\frac{V}{C_{1}}\right)^{k_{1}}\right) \cdot d V
$$

s'écrit sous la forme :

$$
\mathrm{I}_{2}=P_{n} \cdot\left(F\left(V_{c}\right)-F\left(V_{n}\right)\right)
$$


Donc l'intégrale (10) s'écrit sous la forme suivante :

$\bar{P}=\int_{V_{d}}^{V_{n}^{n}}\left(\alpha+\beta \cdot V+\gamma \cdot V^{2}\right) \cdot \frac{k_{1}}{C_{1}} \cdot\left(\frac{V}{C_{1}}\right)^{k_{1}-1} \cdot \exp \left(-\left(\frac{V}{C_{1}}\right)^{k_{1}}\right) \cdot d V+P_{n} \cdot\left[\exp \left[-\left(\frac{V_{n}}{C_{1}}\right)^{k_{1}}\right]-\exp \left[-\left(\frac{V_{c}}{C_{1}}\right)^{k_{1}}\right]\right]$

Le deuxième terme de l'intégrale se calcule plus facilement tandis que le premier terme, par contre plus complexe, nécessite une résolution par une méthode d'intégration numérique.

\section{2-2. Facteur de puissance}

Le facteur de puissance d'un système de conversion de l'énergie éolienne W.E.C.S (Wind Energy Conversion System) défini par le rapport de la puissance produite dans un régime de vent donné par sa puissance nominale (maximale) est représenté sous la forme suivante :

$$
F_{P}=\left(\frac{\bar{P}}{P_{n}}\right)
$$

avec:

$\bar{P}$ : Puissance moyenne annuelle fournie par l'aérogénérateur [Watt],

$P_{n}$ : Puissance nominale de l'aérogénérateur [Watt].

La production de l'électricité par l'aérogénérateur et le moteur Diesel est un compromis entre l'aspect technique et économique, donc il est important de déterminer et de minimaliser le coût de production du Kwh ; pour le moteur Diesel il est influencé par le prix du combustible (mazout) et les frais de maintenance pour tout le système hybride.

Les Figures 1 et 2 montrent que le facteur de puissance et la puissance moyenne annuelle de sortie augmentent avec la hauteur jusqu'à aboutir à une stabilité.

\section{Résultats et interprétation}

Le prix du Kwh est donné par la formule suivante :

$$
P=\frac{F_{1}}{d} \frac{\psi}{W}+\frac{y_{B}}{\eta} \frac{F_{2}}{d w}+F_{3}
$$

avec:

$F_{1}$ : Frais installation du moteur Diesel (DA).

$\mathrm{F}_{2}$ : Frais d'exploitation (DA).

$\mathrm{F}_{3}$ : Frais d'exploitation variables (DA/Kwh). 
D : Durée annuelle d'utilisation équivalente.

W : Puissance nominale.

$\psi$ : Facteur d'annuité.

$$
\psi=\frac{q^{n(q 1)}}{q^{n} 1}
$$

avec: $\quad q=1+\frac{Z}{100}$

$Z$ : Taux d'intérêt.

$N$ : Taux d'amortissement.

$Y_{B}$ : Prix du carburant (DA/Kwh).

$\eta$ : Rendement de la productivité d'électricité.

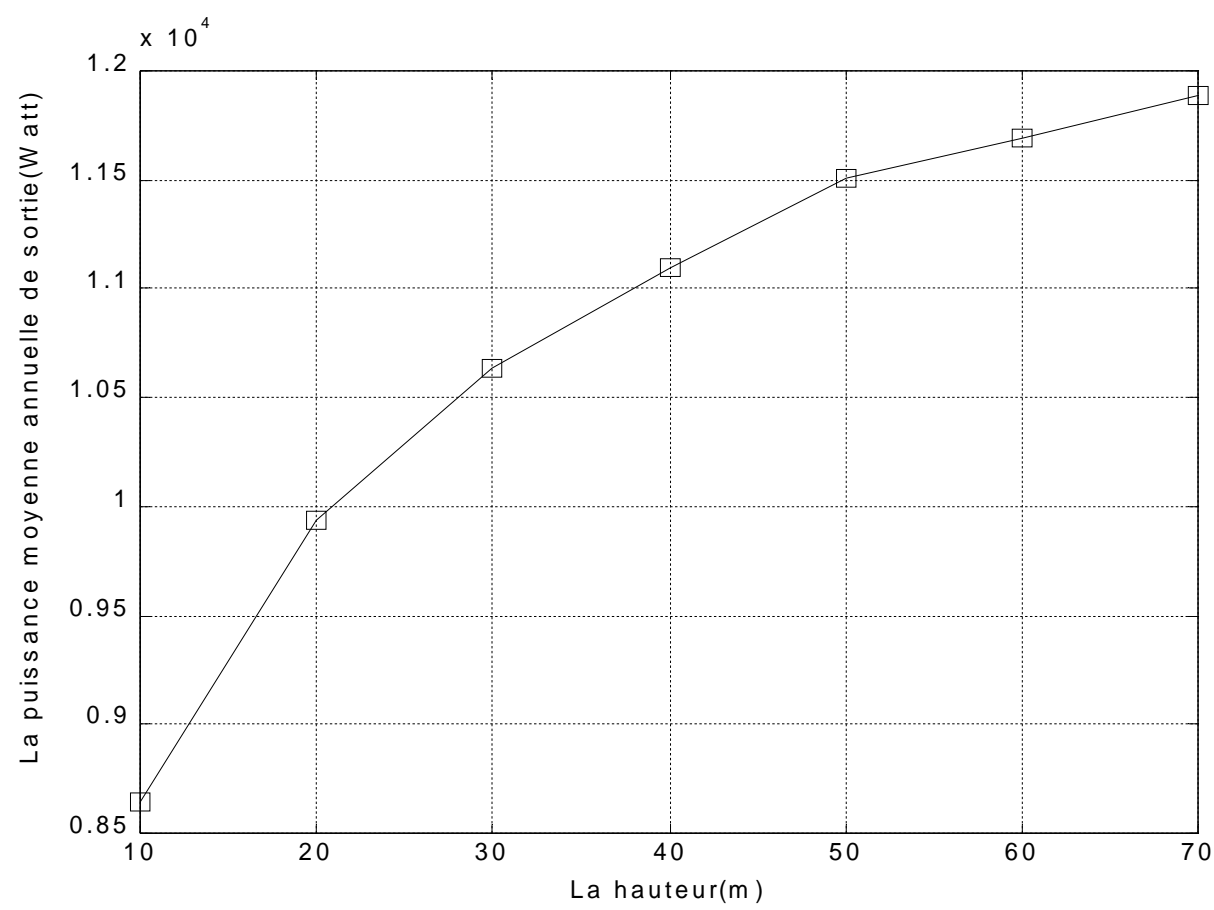

Figure 1 : Variation de la puissance moyenne annuelle de sortie en fonction de la hauteur 


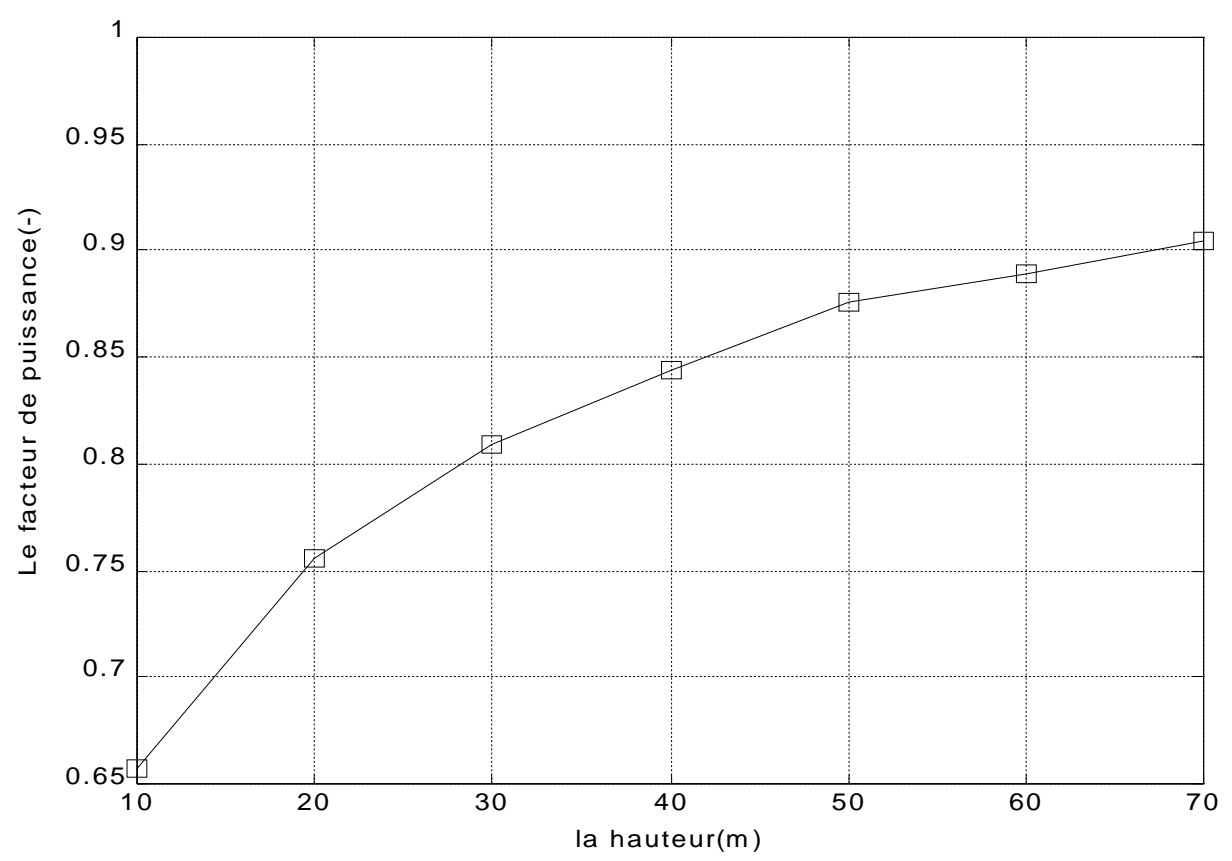

Figure 2 : Variation du facteur de puissance en fonction de la hauteur

Ainsi le système choisi aura une puissance nominale de 1500 Watt. À 20 mètres a.d.s, il pourra alimenter 13 foyers/an (Tableau I) sachant qu'un foyer en Algérie consomme en moyenne $2 \mathrm{kWh} /$ jour $[6,7,8]$.

Les résultats montrent que le nombre de foyers alimenté sera plus important si la hauteur augmente, ce qui entraîne en même temps une augmentation de la puissance moyenne de sortie de l'aérogénérateur. Ainsi le système retenu pourra être installé à une hauteur de 25 mètres a.d.s et alimentera dans ces conditions 14 foyers/an, la puissance moyenne annuelle de sortie étant de $10303 \mathrm{kWh} / \mathrm{an}$.

Tableau 1 : Nombre de foyers pouvant être alimenté en fonction de la hauteur par un système de 1500 Watt.

\begin{tabular}{|c|c|c|c|c|c|c|c||}
\hline Site & \multicolumn{7}{|c|}{ Tlemcen } \\
\hline Hauteur (m) & 10 & 20 & 30 & 40 & 50 & 60 & 70 \\
\hline Nombre de foyers & 11 & 13 & 14 & 15 & 15 & 16 & 16 \\
\hline
\end{tabular}

Dans notre cas on a retenu un aérogénérateur de 1500 Watt pour un besoin moyen (alimentation de 14 foyers de consommation moyenne), le prix de la machine étant 
estimé à $575000 \mathrm{DHT}$, les travaux de génie civil, et de pose de raccordements (5 à 6 jours) estimé à $287500 \mathrm{DHT}$, le parc de stockage estimé à $287500 \mathrm{DHT}$, le régulateur et l'onduleur estimés à $172500 \mathrm{DHT}$, le récepteur adapté estimé à $345000 \mathrm{DHT}$, soit un total de 1667500 DHT, qui représente le coût total de l'investissement $[9,10,11]$.

L'entretien des machines tournantes est primordial pour leur bon fonctionnement et leur longévité. Une visite annuelle est conseillée pour la vérification des roulements, (graissage).

On estime que l'entretien de l'aérogénérateur représente $1 \%$ de l'investissement correspondant à sa première année de fonctionnement.

\section{3-1. Rentabilité de l'investissement}

En général un investissement dont la rentabilité est grande s'avérera encore plus rentable après impôts.

S'il en est ainsi, c'est que la réglementation des amortissements pour toute sorte d'affaires tend à être très favorable dans la plupart des pays. Avec des amortissements fiscaux rapides, on peut amortir la dépréciation de l'actif plus vite qu'il ne perd réellement de sa valeur, sachant que le taux de rendement de l'investissement augmente [12].

Les fluctuations du DINAR nous amènent à calculer le taux d'intérêt par la relation suivante :

$$
\tau=\frac{1}{(1+t)}
$$

avec :

$t$ : Taux d'intérêt annuel $5 \%$ /an.

Taux d'inflation en Algérie est $20 \%$ /an.

$$
\zeta=\frac{(1+t)}{\left(1+i_{1}\right)}
$$

avec: $i_{1}$ : Taux d'inflation est $20 \%$,

$\zeta$ : Rentabilité réelle de l'énergie éolienne [-].

Donc on calcule notre rentabilité d'investissement sur 20 ans.

On remarque d'après la courbe (Figure 3) que l'énergie éolienne est intéressante à long terme; au début l'investissement de l'installation est élevé puis devient rentable dans le temps. 


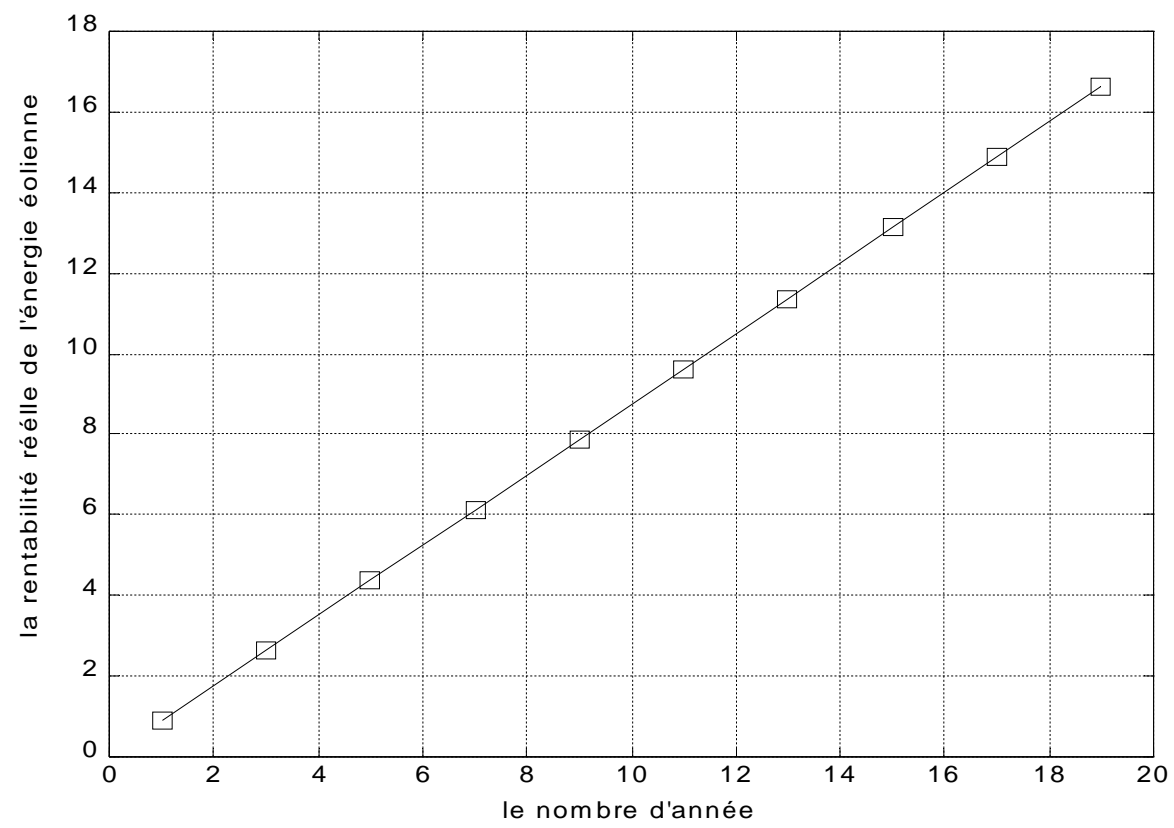

Figure 3 : Rentabilité réelle de l'énergie éolienne en fonction du nombre d'années

\section{Conclusion}

En conclusion, on constate que le potentiel énergétique récupérable au site de Tlemcen (Aéroport) atteint les $425.16 \mathrm{kWh} / \mathrm{m}^{2}$.an. En effet l'asymétrie de la distribution entraîne une variation particulière de chaque site traité, et pour connâitre le potentiel énergétique éolien d'un site quelconque il est impératif de passer par l'extrapolation verticale des paramètres de Weibull qui renseignent sur la densité de puissance moyenne annelle; dans notre cas, cette dernière a été estimée à $607.99 \mathrm{kWh} / \mathrm{m}^{2}$.an à une hauteur optimale de 25 mètres a.d.s.

Donc pour le dimensionnement d'un aérogénérateur, il est impératif de passer par l'étude statistique des vents sur le site considéré afin de déduire le potentiel éolien.

En effet l'asymétrie de la distribution pour chaque site traité permet d'estimer la puissance de sortie d'un aérogénérateur et ceci après modélisation de la distribution du vent par la loi de Weibull. Aussi, la puissance moyenne annuelle et le facteur de puissance augmentant avec la hauteur, les aérogénérateurs fonctionneront avec une très grande efficacité à des hauteurs plus élevées. Ce travail nous a permis d'avoir une idée sur les possibilités d'alimentation en énergie électrique des localités isolées, éloignées, là où il ne peut y avoir de raccordements de câbles électriques à cause de leur coût de 
revient élevé.

Dans notre étude, nous nous sommes intéressés à la rentabilité de l'investissement de l'installation de l'aérogénérateur sur 20 ans en nous basant sur un taux d'intérêt annuel de $5 \%$ /an et un taux d'inflation de $20 \%$ /an. On constate que l'installation impose un investissement élevé la première année (travaux d'installation) et commence à être rentable après quelques années, ce qui entraîne la diminution du prix de revient du Kilowattheure, jusqu'à devenir compétitif.

\section{Références}

[1] - R. DUMON, "Economies et conversion d'énergie", Ed. Masson, Paris, (1978)

[2] - R. DAVID, que sais je "La conversion des énergies", Presses Universitaires de France, Paris, (1966)

[3] - M. DAHLGREN, "Wind former", A.B.B Corporate Research 3, Mars (2000), pp. $31-37$

[4] - D. LE GOURIERES, "Energie éolienne", Théorie Conception et Calcul Pratique des Installations, Ed. Eyrolles, Paris, (1982)

[5] - C. G. JUSTUS, traduit et adapté par J. L Plazy, "Vent et performances des éoliennes", Ed. S C M, Paris, (1982)

[6] - L. HAMMANE, A. KHELLAF, et N. AIT. MESSAOUDENE, II Estimation de la puissance

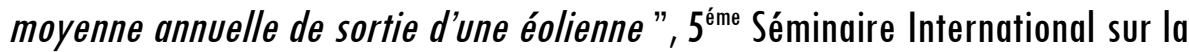
Physique Énergétique, Bechar, Algérie, 07 au 09 Novembre (2000), pp. 6-13

[7] - E. H. LYSEN, "Introduction to Wind Energy", Ed. S.W.D, $2^{\text {éme }}$ Ed, the Netherlands, May, (1983)

[8] - 0. GUERRI, "Etude des performances et des systèmes d'application de deux éoliennes à axe vertical ", Thèse de Magister, Centre de Développement des Énergies Renouvelables (C.D.E.R), Alger, Juillet (1986)., Université des Sciences et de la Technologie (USTHB)

[9] - J. HASLETT, " The capacity credit of wind power : A theoretical analysis", Solar Energy, Vol. 26, January (1981), pp. 391-401

[10] - N. KASBADJI MERZOUK, M. MERZOUK et N. MESSEN, "Mass consistent model application to the desertification phenomena study in the high plains of Algeria", International Journal of Renewable Energy, $\mathrm{N}^{\circ} 28$ (2003) pp. 655-663

[11] - A. BOUHANIK, "Bulletin mensuel météorologique et radiométrique", Bouzaréah, septembre (2003)

[12] - N. KASBADJI MERZOUK et M. MERZOUK, "Usefull power density of a wind machine in Algeria", Proceeding of international mechanical engineering conference, $\mathrm{Ko}$ weit, (2004) 